\title{
ZOOPHAGIE ET HÔtES ALTERNATIFS DES VECTEURS DU PALUDISME AU SÉNÉGAL
}

\author{
KONATÉ L.*, FAYE O.*, GAYE O.**, SY N.*, DIOP A.*, DIOUF M.*, TRAPE J-F.*** \& MOLEZ J.-F.***
}

Summary : OBSERVATIONS ON THE FEEDING PATTERNS AND THE ALTERNATIVE HOSTS SELECTION OF THE MALARIA VECTORS IN SENEGAL

Blood-engorged females of An. gambiae s.l., An. funestus, An. pharoensis and An. rufipes caught resting indoors were tested (precipitin or enzyme-linked immunosorbent assay) to determine the source of bloodmeal. The species of the An. gambiae complex fed mainly on human hosts in all prospected areas, except in those of the mid Senegal river valley where an important zoophily was observed within and near the irrigation zone. Among 4,597 blood-engorged females of An. gambiae s.l., $29 \%$ fed on cattle with $7,5 \%$ of mixed boodmeals, mainly human-bovine or humanequine. The presence of cattle, culicid population densities and individual mosquito safety devices were the most determinant factors of animal deviation. Blood of most domestic animals was found in the stomach of collected females, but according to areas, bovines and equines were the main hosts for zoophilic females of An. gambiae s.l. Females of An. funestus collected in the middlewest were more anthropophilic than those collected in southeastern areas. An. pharoensis was most prevalent in the Senegal river delta where it was found to be very anthropophilic. An. rufipes was strongly endophilic but exclusively zoophilic in all prospected areas.

KEY WORDS : vectors, malaria, anthropophily, zoophily, Senegal.

\section{Résumé :}

L'origine des repas sanguins des femelles d'Anopheles gambiae s.l., d'An. funestus, d'An. pharoensis et d'An. rufipes a été déterminée par tests enzymatiques (précipitines ou ELISA) dans les différentes zones bioclimatiques du Sénégal. Les espèces du complexe An. gambiae se sont montrées très polyphages, mais se nourrissent préférentiellement sur homme dans toutes les localités prospectées. Des 4597 femelles endophiles d'An. gambiae s.l. collectées, $29 \%$ étaient gorgées sur animal avec 7,5\% de repas mixtes dont principalement des repas homme-bovin et hommeéquin. Une importante déviation animale a été observée dans les localités proches des aménagements agricoles irrigués de la moyenne vallée du fleuve Sénégal. La présence d'animaux, hôtes alternatifs, la densité des populations culicidiennes et

l'inaccessibilité des hôtes humains semblent être les facteurs les plus déterminants de cette déviation animale. Le sang des différents animaux domestiques a été retrouvé dans l'estomac des moustiques récoltés, mais les bovidés et équidés ont été les principaux hôtes pour les femelles zoophages. An. funestus, moins répandu que An. gambiae s.l., a été significativement plus zoophage dans le sud-est que dans le centre-ovest du pays. An. pharoensis, particulièrement abondant dans le delta du fleuve Sénégal, se nourrit essentiellement sur homme dans toutes les régions prospectées. An. rufipes, espèce très endophile, est strictement zoophage dans toutes les localités où il est rencontré

MOTS CLÉS : vecteurs, paludisme, anthropophilie, zoophilie, Sénégal.

\section{INTRODUCTION}

$\mathrm{D}$ ans le paludisme humain, la compétence vectorielle d'une espèce culicidienne dépend essentiellement de sa longévité et de son comportement trophique. Les préférences trophiques des moustiques, et des anophèles en particulier, ont fait l'objet de nombreuses études en Afrique (Bruce-Chwatt et al., 1960, 1966; Hamon et al., 1964; Hamon et al., 1965; Chandler et al., 1975; Carnevale \& Boreham, 1978; Snow, 1983; Beier et al., 1988; Gordon et al.,

\footnotetext{
* Département de Biologie Animale, Faculté des Sciences et Techniques, Université Cheikh Anta Diop, BP 5005, Dakar, Sénégal.

** Service de Parasitologie, Faculté de Médecine et de Pharmacie, Université Cheikh Anta Diop, BP 5005, Dakar, Sénégal.

${ }^{* * *}$ Laboratoire de Paludologie, Centre IRD-Orstom, BP 1386, Dakar, Sénégal.

Correspondance : Lassana Konaté.

Tél. : (221) 252530 - Fax : (221) 252529.
}

1991; Sharp \& Le Sueur, 1991; Mnzava et al., 1995; Lemasson et al., 1997). Les premières étaient basées sur l'observation directe, l'attractivité d'hôtes potentiels utilisés comme appâts ou l'examen cytologique du repas sanguin. L'étude de l'indice d'anthropophilie s'est développée avec le test des précipitines (Schubert \& Holdeman, 1956; Tempelis \& Lofy, 1963). Cet outil sérologique permettait de préciser l'origine des repas de sang des arthropodes hématophages. Actuellement, la technique ELISA (Enzyme Linked Immunosorbent Assay), du fait de sa plus grande sensibilité, reste la plus employée à cet effet (Beier et al., 1988).

Les études entomologiques réalisées dans les différentes régions du Sénégal (fig. 1) ont révélé l'hétérogénéité du comportement trophique des vecteurs de paludisme selon les zones bioclimatiques (Vercruysse \& Jancloes, 1981; Vercruysse, 1985; Petrarca et al., 1987; Konaté et al., 1994a; Konaté et al., 1994b; Faye et al., 1993; Faye et al., 1994; Faye et al., 1995a; Faye 

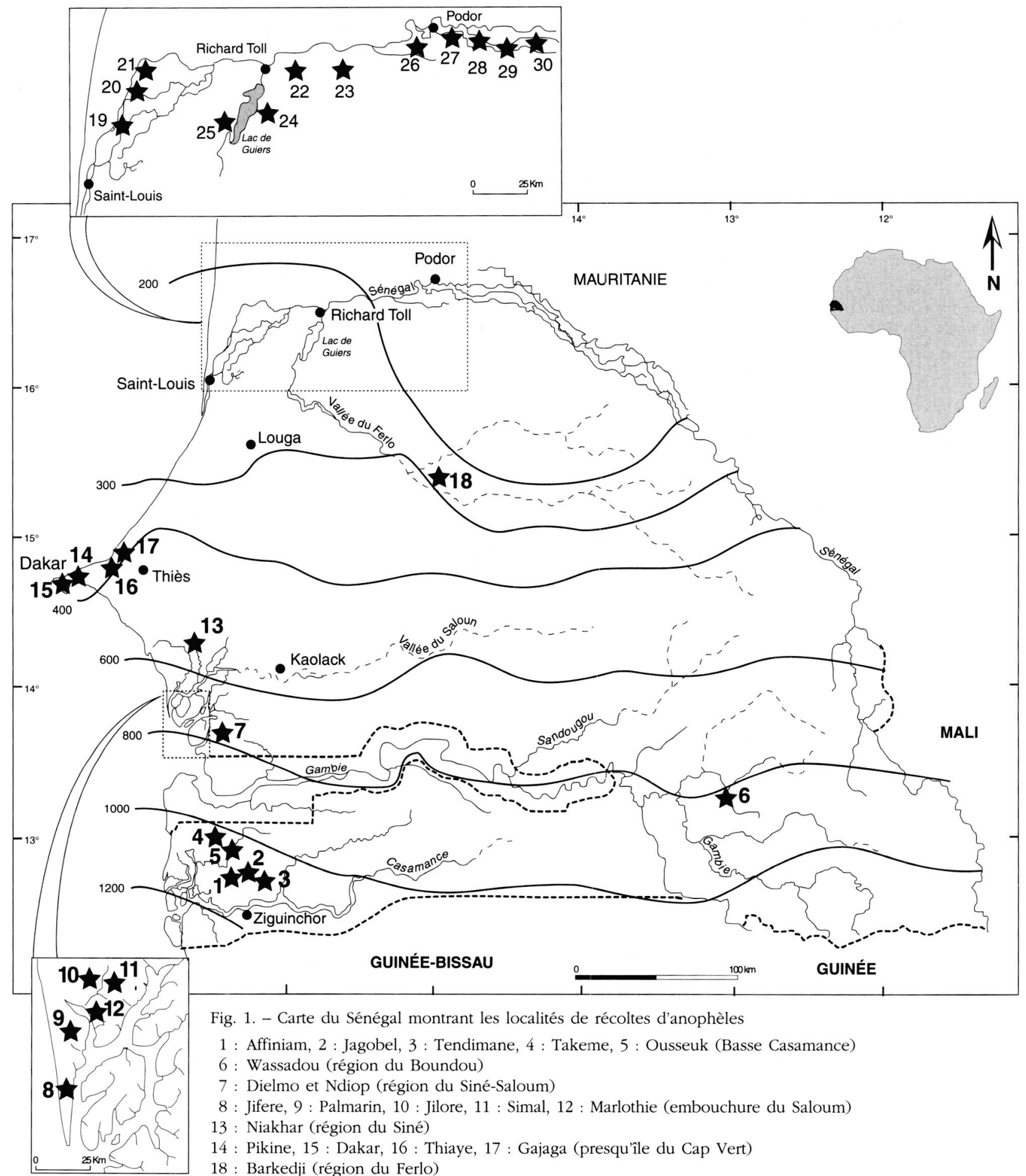

Fig. 1. - Carte du Sénégal montrant les localités de récoltes d'anophèles

1: Affiniam, 2 : Jagobel, 3 : Tendimane, 4 : Takeme, 5 : Ousseuk (Basse Casamance)

6: Wassadou (région du Boundou)

7: Dielmo et Ndiop (région du Siné-Saloum)

8: Jifere, 9: Palmarin, $10:$ Jilore, 11: Simal, 12: Marlothie (embouchure du Saloum)

13: Niakhar (région du Siné)

14 : Pikine, 15 : Dakar, 16 : Thiaye, 17 : Gajaga (presqu'île du Cap Vert)

18: Barkedji (région du Ferlo)

19: Maka, 20 : Boundoum, 21 : Kassak (delta du fleuve Sénégal)

22: Keur Mbaye, 23 : Ndiareme (basse vallée du fleuve)

24: Mbane, 25: Nder (région du lac de Guiers)

26 : Aere Lao, $27:$ Boke Jaobe, $28:$ Jomandou Walo, $29:$ Jomandou Jeri, $30:$ Toulde Galle (moyenne vallée du fleuve) 
et al., 1995b) et selon les localités d'une même zone (Mbaye, 1997; Faye, 1997). La diversité des situations est, pour une large part, déterminée par le niveau d'anthropophilie des vecteurs, traduisant leurs contacts avec les humains, sujets infectants ou susceptibles d'être infectés. Les spécificités locales, inéluctables aux études de transmission palustre, rendent difficiles les comparaisons inter- et intra-zonales. Ces dernières restent cependant nécessaires. En effet, l'étude du comportement trophique des vecteurs n'a d'intérêt que pour comparer différentes situations afin d'en tirer des informations épidémiologiques utiles. Ce travail présente une analyse des différentes observations sur le comportement trophique d'Anopheles gambiae s.l., d'An. funestus (vecteurs majeurs de paludisme au Sénégal) et d'espèces associées (An.pharoensis, An. rufipes).

\section{ANOPHELES GAMBIAE S.L.}

\section{TROPISME ET INDICE D'ANTHROPOPHILIE}

Au Sénégal, la transmission du paludisme est exclusivement (Vercruysse \& Jancloes, 1981; Vercruysse, 1985; Petrarca et al., 1987; Faye, 1997; Faye et al., 1993, 1994; 1995a, 1995b, 1995c; Konaté et al., 1994b; Robert et al., 1997; Mbaye, 1997) ou en grande partie (Konaté et al., 1994a; Fontenille et al., 1997a) assurée par les espèces du complexe An. gambiae. L'étude de l'indice d'anthropohilie (IA) des femelles d'An. gambiae s.l. (tableaux I et II), montrent qu'elles se nourrissent largement sur homme dans toutes les localités prospectées, excepté dans la moyenne vallée du fleuve Sénégal (zone sahélienne).

Cette observation peut s'expliquer en partie par un biais d'échantillonnage. En effet dans les études sur la dynamique de transmission palustre, la récolte de faune anophélienne endophile (faune résiduelle dans les chambres) est préférentiellement ciblée. Même si on note davantage de moustiques gorgés sur homme dans les chambres à coucher et une majorité de

\begin{tabular}{|c|c|c|c|}
\hline Zones/Localités & $\begin{array}{l}\text { Nombre } \\
\text { de repas }\end{array}$ & IA & Références \\
\hline \multicolumn{4}{|l|}{ Zone guinéenne } \\
\hline Affiniam-Jagobel-Tendimane & 375 & 0,845 & Faye et al., 1994 \\
\hline Takeme-Ousseuk & 182 & 0,896 & " \\
\hline Wassadou & 945 & 0,898 & Faye et al., 1995a \\
\hline Sous-total & 1502 & 0,884 & \\
\hline \multicolumn{4}{|l|}{ Zone soudanienne } \\
\hline Dielmo & 208 & 0,644 & Konaté et al., 1994a \\
\hline Jifere & 806 & 0,979 & Faye, 1997 \\
\hline Palmarin & 143 & 0,937 & " \\
\hline Jilore & 96 & 0,833 & " \\
\hline Simal & 411 & 0,796 & “ \\
\hline Marlothie & 165 & 0,915 & " \\
\hline Niakhar & 771 & 0,829 & Robert et al., 1998 \\
\hline Dakar & 43 & 0,977 & Konaté et al., 1994b \\
\hline Thiaye-Gajaga & 443 & 0,756 & Faye et al., 1995b \\
\hline Sous-total & 3086 & 0,853 & \\
\hline \multicolumn{4}{|l|}{ Zone sahélienne } \\
\hline Maka-Boundoum-Kassak & 32 & 0,719 & Faye et al., 1995c \\
\hline Keur Mbaye & 438 & 0,683 & Mbaye, 1997 \\
\hline Ndiareme & 372 & 0,419 & " \\
\hline Mbane & 29 & 0,586 & " \\
\hline Nder & 51 & 0,882 & “ \\
\hline Aere Lao & 157 & 0,630 & Vercruysse, 1985 \\
\hline Boke Jaobe & 55 & 0,454 & " \\
\hline Jomandou Walo & 357 & 0,437 & Faye et al., 1993 \\
\hline Jomandou Jeri & 163 & 0,619 & " \\
\hline Toulde Galle & 103 & 0,777 & $"$ \\
\hline Sous-total & 1757 & 0,569 & \\
\hline
\end{tabular}

Tableau I. - Récapitulatif du nombre de repas sanguins analysés et indice d'anthropophilie (IA) des femelles d'An. gambiae s.l. récoltées dans différentes régions du Sénégal (1985-1997)*.

* L'étude n'a porté que sur les seules femelles endophiles qui ont été échantillonnées par des récoltes après pulvérisations intradomiciliaires de pyréthrinoïdes. Les différentes espèces anophéliennes ont été identifiées d'après leurs caractères morphologiques. La détermination de l'origine des repas sanguins a été faite par la méthode des précipitines pour les récoltes en Basse Casamance et par ELISA dans les autres localités.

\begin{tabular}{|c|c|c|c|c|c|c|c|}
\hline \multirow{2}{*}{$\begin{array}{c}\text { Repas } \\
\text { de sang analysés } \\
\text { Localités ou régions }\end{array}$} & \multirow{2}{*}{$\begin{array}{c}\text { Total } \\
\text { repas de sang } \\
\text { analysés } \\
\mathrm{N}\end{array}$} & \multicolumn{2}{|c|}{$\begin{array}{l}\text { Repas simple : } \\
\text { sang humain }\end{array}$} & \multicolumn{2}{|c|}{$\begin{array}{c}\text { Repas mixte : } \\
\text { humain/animal }\end{array}$} & \multicolumn{2}{|c|}{$\begin{array}{l}\text { Repas animaux : } \\
\text { (1 ou } 2 \text { hôtes) }\end{array}$} \\
\hline & & $\mathrm{N}$ & $(\%)$ & $\mathrm{N}$ & (\%) & $\mathrm{N}$ & $(\%)$ \\
\hline Wassadou & 944 & 834 & $(88,3)$ & 14 & $(1,5)$ & 96 & $(10,2)$ \\
\hline Embouchure du Saloum & 1612 & 1327 & $(82,3)$ & 145 & (9) & 140 & $(8,7)$ \\
\hline Niayes & 486 & 357 & $(73,5)$ & 20 & $(4,1)$ & 109 & $(22,4)$ \\
\hline Moyenne vallée du fleuve & 620 & 284 & $(45,9)$ & 50 & (8) & 286 & $(46,1)$ \\
\hline Basse vallée du fleuve & 919 & 473 & $(51,5)$ & 66 & $(7,2)$ & 380 & $(41,3)$ \\
\hline Total & 4581 & 3275 & $(71,5)$ & 295 & $(6,4)$ & 1011 & $(22,1)$ \\
\hline
\end{tabular}

Tableau II. - Analyse des repas sanguins (par ELISA) chez An. gambiae s.l. et détail des repas simples (humain ou animal) et des repas mixtes (humain/animal) selon les localités au Sénégal (1993-1997). 
femelles nourries sur bétail dans les étables, débarras et abris annexes, une faible proportion de repas hétérologues est toujours retrouvée (Hamon et al., 1964; Konaté et al., 1994a).

\section{Zone sahélienne}

Les moyenne et basse vallées et le delta du fleuve Sénégal sont situés dans les zones sahélienne (située au dessus de l'isohyète $200 \mathrm{~mm}$ ) et soudano-sahélienne (entre 200 et $400 \mathrm{~mm}$ ). Depuis la mise en service du barrage de Diama dans le delta, de nombreux périmètres irrigués villageois ont été réalisés dans la moyenne et basse vallée et le delta. De ce fait, la riziculture est devenue la principale activité agricole dans la région, avec, comme conséquence majeure, une multiplication des gîtes préimaginaux et un allongement de la période de production anophélienne dans les zones d'aménagement hydroagricole. Dans la vallée du fleuve Sénégal, l'IA d'An. gambiae s.l. est variable en fonction de la localité de collecte des femelles. Il a été en moyenne plus faible en zone de riziculture irriguée qu'en zone de culture pluviale, aussi bien dans la moyenne vallée (Jomandou Walo et Jomandou Jeri vs Toulde Galle) que dans la basse vallée (Keur Mbaye et Ndiareme vs Mbane/Nder) du fleuve Sénégal (Faye et al., 1993; Mbaye, 1997). Dans le delta, la faiblesse des effectifs d'An. gambiae s.l. n'autorise pas la comparaison entre localités de zones avec et sans aménagements hydro-agricoles.

\section{Zone soudanienne}

La zone soudanienne est située entre les isohyètes $400 \mathrm{~mm}$ et $800 \mathrm{~mm}$ (soudano-guinéenne entre 800 et $1000 \mathrm{~mm}$ ). Dans la presqu'île du Cap-Vert, les IA enregistrés en milieu urbain (Dakar et Pikine) sont plus élevés que ceux enregistrés en milieu rural, dans deux petits villages de la zone des Niayes (Thiaye et Gajaga). L'IA moyen d'An. gambiae s.l. a été significativement plus faible dans les régions du Sine (Niakhar) et du Saloum (Dielmo) que dans les villages côtiers de l'estuaire du Saloum (Jifere, Palmarin, Jilore, Simal et Marlothie) où il a varié en fonction de la localité de collecte des femelles comme l'avaient rapporté BruceChwatt et al. (1960).

\section{Zone guinéenne}

Dans cette zone située en dessous de l'isohyète $1000 \mathrm{~mm}$, seules des localités du littoral ont été prospectées. Il a été constaté que l'IA des femelles d'An. gambiae s.l. récoltées dans les villages proches (Affiniam, Tendimane et Jagobel) et éloignés (Takeme et Ousseuk) de la mangrove ont été comparables (Faye et al., 1994).

\section{TROPISME ANIMAL ET ZOOPHAGIE}

Parmi les 4581 repas sanguins de femelles d'An. gambiae s.1. analysés (tableaux II, III et IV), 22 \% (1011) avaient été prélevés sur animal. L'identification des repas animaux a montré que 977 étaient pris sur un hôte unique et que 339 étaient des repas mixtes animal/homme ou animal/animal (parmi ces derniers, trois repas avaient été pris sur trois hôtes différents). Les repas mixtes contenant du sang humain prédominaient dans toutes les localités. Pour les repas provenant de deux espèces animales distinctes, les associations équin/ovin, bovin/ovin et équin/bovin étaient les plus fréquentes; les autres types d'associations étaient plus rares. Les femelles zoophages d'An. gambiae s.l. ont donc présenté deux profils d'alimentation animale : celui où les bovidés constituent la principale source des repas sanguins et celui où les équidés ont été les principaux hôtes.

\section{Zone d'élevage}

En milieu rural du Sénégal, l'élevage est en général de type traditionnel et son importance est variable selon les ethnies, les localités et les zones géographiques. De façon générale, les régions du centre (Ferlo) et du sudest (Boundou) sont de grandes zones d'élevage. Lorsque les différents animaux domestiques présentent des conditions d'accessibilité similaires (parquage dans des enclos à proximité immédiate des habitations), les bovins constituent les hôtes animaux de premier choix pour les femelles zoophages d'An. gambiae s.l. (tableaux III et IV).

\begin{tabular}{|c|c|c|c|c|c|c|c|c|c|c|c|}
\hline \multirow{3}{*}{$\begin{array}{c}\text { Analyse } \\
\text { des repas de sang } \\
\text { Localités/régions }\end{array}$} & \multicolumn{10}{|c|}{ Catégories de repas mixtes : homme/animal } & \multirow[b]{3}{*}{ Tota } \\
\hline & \multicolumn{2}{|c|}{ Homme/bovin } & \multicolumn{2}{|c|}{ Homme/équin } & \multicolumn{2}{|c|}{$\begin{array}{c}\text { Homme/ } \\
\text { ovin-caprin }\end{array}$} & \multicolumn{2}{|c|}{ Homme/canin } & \multicolumn{2}{|c|}{ Homme/volaille } & \\
\hline & $\mathrm{N}$ & (\%) & $\mathrm{N}$ & $(\%)$ & $\mathrm{N}$ & $(\%)$ & $\mathrm{N}$ & (\%) & $\mathrm{N}$ & $(\%)$ & \\
\hline Wassadou & 3 & $(21,4)$ & 9 & $(64,3)$ & 1 & $(7,1)$ & 0 & & 1 & $(7,1)$ & 14 \\
\hline Embouchure du Saloum & 28 & $(19,3)$ & 67 & $(46,2)$ & 1 & $(0,7)$ & 48 & $(33,1)$ & 1 & $(0,7)$ & 145 \\
\hline Niayes & 7 & (35) & 12 & $(60)$ & 0 & & 0 & & 1 & (5) & 20 \\
\hline Moyenne vallée du fleuve & 46 & (92) & 0 & & 3 & (6) & 0 & & 1 & (2) & 50 \\
\hline Basse vallée du fleuve & 3 & $(4,5)$ & 51 & $(77,3)$ & 12 & $(18,2)$ & 0 & & 0 & & 66 \\
\hline Total & 87 & $(29,5)$ & 139 & $(47,1)$ & 17 & $(5,8)$ & 48 & $(16,3)$ & 4 & $(1,3)$ & 295 \\
\hline
\end{tabular}

Tableau III. - Analyse des repas sanguins (par ELISA) chez An. gambiae s.l. et détail des repas mixtes (humain/animal) selon les localités au Sénégal (1993-1997). 


\begin{tabular}{|c|c|c|c|c|c|c|c|c|}
\hline \multirow{2}{*}{$\begin{array}{c}\text { Analyse } \\
\text { des repas de sang } \\
\text { Localités ou régions }\end{array}$} & \multicolumn{6}{|c|}{ Repas simple : 1 seul hôte animal } & \multirow{2}{*}{$\begin{array}{c}\text { Repas mixtes : } \\
\mathbf{2} \text { hôtes animaux } \\
\text { Espèce A et espèce B } \\
\mathrm{N}\end{array}$} & \multirow[b]{2}{*}{$\begin{array}{c}\text { Grand } \\
\text { total }\end{array}$} \\
\hline & $\begin{array}{c}\text { Bovin } \\
\mathrm{N}\end{array}$ & $\begin{array}{c}\text { Équin } \\
\mathrm{N}\end{array}$ & $\begin{array}{c}\text { Canin } \\
\mathrm{N}\end{array}$ & $\begin{array}{c}\text { Ovin/caprin } \\
\mathrm{N}\end{array}$ & $\begin{array}{c}\text { Volaille } \\
\mathrm{N}\end{array}$ & Total & & \\
\hline Wassadou & 44 & 48 & 0 & 1 & 0 & 93 & 3 & 96 \\
\hline Embouchure du Saloum & 7 & 106 & 17 & 4 & 0 & 134 & 6 & 140 \\
\hline Niayes & 50 & 46 & 0 & 4 & 3 & 103 & 6 & 109 \\
\hline Moyenne vallée du fleuve & 200 & 47 & 0 & 27 & 5 & 279 & 17 & 286 \\
\hline Basse vallée du fleuve 11 & 338 & 0 & 19 & 0 & 368 & 12 & 380 & \\
\hline Total & 312 & 585 & 17 & 55 & 8 & 977 & $44^{*}$ & 1.011 \\
\hline
\end{tabular}

* sur les 44 repas mixtes (deux hôtes) les associations les plus fréquentes étaient : équin/ovin (12), bovin/ovin (11) et équin/bovin (8) et autres associations diverses (13) dont trois repas sur trois hôtes.

Tableau IV. - Analyse des repas sanguins (par ELISA) chez An. gambiae s.l. et détail des repas animaux : un ou deux hôtes (espèces différentes), selon les localités au Sénégal (1993-1997).

Zone d'agriculture pluviale

Les régions de l'ouest du Sénégal et tout le bassin arachidier sont principalement agricoles. Cependant, les villages de ces régions possèdent parfois un cheptel restreint constitué principalement de petits ruminants (ovins et caprins). En l'absence de bovins, ce sont plus fréquemment les équidés et dans une moindre mesure les ovins-caprins qui constituent les sources de repas sanguins des femelles zoophages d'An. gambiae s.l. (tableaux III et IV). Dans les localités de l'estuaire du Saloum (villages de pêcheurs et d'agriculteurs), les équidés ont été les principaux hôtes pour ces dernières.

Zone de riziculture irriguée

Dans la vallée du fleuve Sénégal (Faye et al., 1993; Mbaye, 1997), la zoophagie des femelles d'An. gambiae s.l. est en moyenne plus élevée en milieu de riziculture qu'en zone de culture pluviale (tableau V). Dans les villages de riziculture du delta, les repas animaux provenaient exclusivement de petits ruminants (ovins et caprins) qui, par ailleurs, ont été les seuls hôtes associés dans les repas mixtes. Toutefois, la faiblesse des effectifs limite la portée de cette observation. Dans les localités situées sur la marge du delta, une forte zoophagie a été notée et les équidés ont été les principaux hôtes (tableaux III et IV). Plus en amont, dans la moyenne vallée, les bovidés ont été les principaux hôtes recherchés par les femelles zoophages d'An. gambiae s.l. (tableaux III et IV).

\section{DÉVIATION TROPHIQUE}

\section{Inaccessibilité des hôtes}

Le comportement trophique des femelles d'anophèles a été surtout fonction de la disponibilité des hôtes humain et/ou animal. Dans la moyenne vallée du fleuve Sénégal, une forte déviation animale d'An. gambiae s.l. avait été notée par Vercruysse (1985). Cette observation a été confirmée par Faye et al. (1993) et s'explique par l'usage généralisé des moustiquaires et la présence de nombreux animaux domestiques qui, en lieu et place des hommes inaccessibles, servent de principaux hôtes aux anophèles. A l'inverse, la faiblesse des densités anophéliennes, l'absence d'animaux ou le très fort ratio homme/animal et la faible dispersion des femelles, expliquent le fait que l'homme soit l'hôte exclusif d'An. gambiae s.l. en milieu urbain de Dakar (Konaté et al., 1994b) et de Pikine (Vercruysse \& Jancloes, 1981; Trape et al., 1992). Par contre, en zone rurale des Niayes (localités de Thiaye et de Gajaga) où le bétail est plus abondant, une importante fraction de femelles zoophages a été notée (Faye et al., 1995b).

Mobilité animale et déviation trophique

En zone d'élevage (région du Ferlo et du Boundou en particulier), le cheptel bovin est disséminé dans l'espace rural pendant la saison des pluies. En saison sèche, les animaux sont regroupés autour des villages où se trouvent les points d'eau. Ces mouvements saisonniers du bétail sont à l'origine de la variation saisonnière des proportions des différents types de repas sanguins (tableau V). Ainsi, à Wassadou (Faye et al., 1995a), l'IA d'An. gambiae s.l. est très élevé pendant la saison des pluies lorsque les bovins sont partis pâturer dans des zones éloignées des habitations. Pendant la saison sèche où les animaux sont ramenés vers les villages, l'IA des femelles est significativement plus faible. Nous pensons qu'il en est de même de la variation saisonnière significative de l'IA observée dans les villages pratiquant à la fois l'agriculture et l'élevage, comme le sont Jomandou Walo et Ndiareme. Cependant, dans ces localités rurales mixtes riveraines du fleuve Sénégal, les phases d'éloignement du bétail ne 


\begin{tabular}{|c|c|c|c|c|c|c|}
\hline \multirow{2}{*}{$\begin{array}{c}\text { Localités de récolte } \\
\text { (faune résiduelle } \\
\text { endophile) }\end{array}$} & \multicolumn{3}{|c|}{ Saisons des pluies (juillet-octobre) } & \multicolumn{3}{|c|}{ Saison sèche (novembre-juin) } \\
\hline & $\mathrm{N}^{\circ}$ & $\mathrm{H}^{*}$ & IA & $\mathrm{N}^{\circ}$ & $\mathrm{H}^{*}$ & IA \\
\hline Wassadou $^{\dagger}$ & 448 & 413 & 0,922 & 497 & 436 & 0,877 \\
\hline Jifere & 327 & 314 & 0,960 & 479 & 475 & 0,992 \\
\hline Palmarin & 97 & 89 & 0,917 & 46 & 45 & 0,978 \\
\hline Jilore & 86 & 71 & 0,825 & 10 & 9 & 0,900 \\
\hline Simal & 151 & 123 & 0,814 & 260 & 204 & 0,785 \\
\hline Marlothe & 128 & 118 & 0,922 & 37 & 33 & 0,892 \\
\hline Jomandou Walo ${ }^{\dagger}$ & 232 & 85 & 0,366 & 125 & 71 & 0,568 \\
\hline Jomandou Jeri & 135 & 87 & 0,644 & 28 & 14 & 0,500 \\
\hline Toulde Galle & 72 & 57 & 0,792 & 31 & 23 & 0,742 \\
\hline Thiaye-Gadiaga & 429 & 322 & 0,750 & 14 & 13 & 0,928 \\
\hline Keur Mbaye & 145 & 99 & 0,683 & 293 & 200 & 0,682 \\
\hline Ndiareme $^{\dagger}$ & 187 & 54 & 0,289 & 185 & 102 & 0,551 \\
\hline
\end{tabular}

$\mathrm{N}^{\circ}=$ nombre de repas analysés par ELISA; $\mathrm{H}^{*}=$ nombre de repas avec sang humain; ${ }^{\dagger}=$ variations significatives : Wassadou $(\mathrm{P}<0,05)$, Jomandou Walo $(\mathrm{P}<0,001)$, Ndiareme $(\mathrm{P}<0,001)$.

Tableau V. - Variations saisonnières de l' IA chez An. gambiae s.l dans différentes localités du Sénégal (1993-1997).

\begin{tabular}{|c|c|c|c|c|c|c|}
\hline \multirow{2}{*}{$\begin{array}{l}\text { Espèces anophèles } \\
\text { Repas de sang } \\
\text { Localités ou régions }\end{array}$} & \multicolumn{2}{|c|}{ An. gambiae s.s. } & \multicolumn{2}{|c|}{ An. arabiensis } & \multicolumn{2}{|c|}{ An. melas } \\
\hline & $\begin{array}{c}\mathrm{Nb} \text { repas } \\
\text { analysés }\end{array}$ & $\begin{array}{c}\text { Sang } \\
\text { humain }\end{array}$ & $\begin{array}{c}\mathrm{Nb} \text { repas } \\
\text { analysés }\end{array}$ & $\begin{array}{c}\text { Sang } \\
\text { humain }\end{array}$ & $\begin{array}{c}\mathrm{Nb} \text { repas } \\
\text { analysés }\end{array}$ & $\begin{array}{c}\text { Sang } \\
\text { humain }\end{array}$ \\
\hline Moyenne vallée du fleuve & 69 & 66 & 96 & 139 & 59 & 55 \\
\hline Wassadou ${ }^{\circ}$ & 69 & 66 & 96 & 85 & - & - \\
\hline Embouchure du Saloum* & 3 & 3 & 19 & 18 & 59 & 55 \\
\hline Niayes & 1 & 1 & 176 & 139 & - & - \\
\hline Moyenne vallée du fleuve & 12 & 5 & 8 & 4 & - & - \\
\hline
\end{tabular}

* = l'identification des espèces du complexe An. gambiae a été faite par PCR.

${ }^{\circ}=$ An. gambiae et An. arabiensis sont sympatriques (Faye et al., 1995a)

* = An. gambiae, An. arabiensis et An. melas sont sympatriques (Faye, 1997).

Tableau VI. - Proportion de repas sanguins (analysés par ELISA) pris sur homme sur un échantillonnage d'espèces du complexe An. gambiae $\left.{ }^{\ddagger}{ }^{\ddagger}\right)$ dans les différentes zones de récolte au Sénégal (1993-1997).

sont pas liées à la disponibilité des points d'eau mais au cycle de la riziculture.

Zoophagie des espèces du complexe gambiae

Les caractères morphologiques (Gilies \& De Meillon, 1968) ne permettent pas d'identifier des espèces anophéliennes appartenant à un complexe. Dans le cas d'An. gambiae s.l., l'emploi de la méthode cytogénétique (Coluzzi et al., 1979; Vercruysse, 1985) et/ou de la PCR (Faye et al., 1993) a montré que la distribution et les proportions des différentes espèces sont variables selon les zones géographiques.

Les variations saisonnières et annuelles de la proportion d'An. gambiae s.s. et d'An. arabiensis ont été bien étudiées dans les villages de Dielmo (Fontenille et al., 1997a), Ndiop (Fontenille et al., 1997b) et Barkedji (Lemasson et al., 1997). Ces études, ainsi que celle de Diatta et al. (1998) ont montré que lorsqu'ils étaient capturés au même moment, à la même place et par la même méthode d'échantillonnage, An. gambiae et An. arabiensis présentent un comportement trophique similaire. En effet, l'indice d'anthropophilie, la pro- portion de repas mixtes ainsi que celle des femelles gorgées sur bétail ont été comparables aussi bien à Ndiop (Fontenille et al., 1997b), Barkedji (Lemasson et al., 1997) qu'à Dielmo (Diatta et al., 1998). Cette observation a été refaite dans différentes localités (tableau VI), mais elle n'est pas vérifiée dans la moyenne vallée du fleuve lorsque An. gambiae et An. arabiensis ne coexistent pas.

Les différentes espèces du complexe An. gambiae qui ont leur gîte en eau douce sont très anthropophiles. Par contre An. melas, inféodé aux zones à eau saumâtre, est bien connu pour sa propension à piquer le bétail (bien qu'il ne dédaigne pas l'homme). Son IA n'était pas connu sur le littoral du Sénégal où il est très répandu sur tout le littoral. Dans l'estuaire du Saloum (Diop, 1998), les villages de Palmarin et de Jifere ont un peuplement anophélien composé à majorité d'An. melas avec 86 à $95 \%$ des anophèles récoltés en faune résiduelle dans les chambres à coucher (contre 0 à $0,2 \%$ d'An. gambiae et 5 à $15 \%$ d'An. arabiensis). Avec une valeur de 0,79 à Palmarin et 0,93 à Jifere (Diop, 1998), l'IA des femelles endo- 
philes d'An. melas est très élevé en région de mangrove, il est comparable à celui d'An. gambiae $(1,0)$ et d'An. arabiensis. $(0,80)$. L'absence de tout bétail dans ces villages, habités exclusivement par des pêcheurs, serait à l'origine de ce comportement opportuniste par une espèce dont la forte tendance zoophile est reconnue (Giglioli, 1964; Bryan, 1979; Snow, 1983).

\section{ANOPHELES FUNESTUS}

An. funestus est avec An. gambiae et An. arabiensis à un moindre degré (indice sporozoïtique habituellement plus bas que celui d'An. gambiae et d'An.funestus) l'un des trois vecteurs majeurs de paludisme au Sénégal et en Afrique. Très anthropophile (White, 1971; White et al., 1972), An. funestus est devenu très rare dans la moitié nord du Sénégal, en raison de la destruction de ses gîtes larvaires par une sécheresse persistante (Faye et al., 1995b). Dans la partie sud du Sénégal, les femelles récoltées en faune résiduelle dans les habitations de Dielmo s'étaient nourries préférentiellement sur homme (Konaté et al., 1994a). Dans ce village, les femelles d'An. funestus ont été significativement moins zoophages que celles d'An. gambiae s.l. Le bovin a été la source de $5 \%$ des 144 repas d'An. funestus analysés (Konaté et al., 1994a) et il a été noté une très faible proportion de femelles zoophages nourries sur ovins-caprins.

Dans le sud-est du Sénégal, les femelles d'An. funestus collectées à Wassadou au Boundou (Faye et al., 1995a) étaient nettement plus zoophages (69,9\% des 61 repas analysés étaient d'origine animale) que celles récoltées à Dielmo dans le Saloum.

\section{ANOPHELES PHAROENSIS}

An. pharoensis est prédominant dans les zones d'aménagement hydroagricoles (riziculture) du delta du fleuve Sénégal. Dans les localités prospectées, $86 \%$ des 544 repas sanguins analysés étaient pris sur homme (Faye et al., 1993; Faye et al., 1995c). Ces résultats confirment les observations de Carrara et al. (1990) malgré des méthodes d'échantillonnage différentes.

Les ovins/caprins ont été la source du repas sanguin chez 10,5\% des femelles zoophages, contre $3 \%$ pour les bovins (16 repas) qui sont rares dans les villages prospectés (villages d'agriculteurs). De même, les équins (deux repas) ne constituent pas un hôte de choix pour les femelles zoophages d'An. pharoensis. A l'instar d'An. gambiae s.l., la zoophagie a été significativement plus faible en zone de cultures pluviales (en marge de la vallée) qu'en zone de riziculture irriguée. Les repas mixtes représentent $4 \%$ du total des repas analysés et dans la moitié des cas (12/24), l'homme était l'un des hôtes associés.

\section{ANOPHELES RUFIPES}

L'analyse des 161 repas sanguins de femelles récoltées à Thiaye, Gajaga, Dielmo, Wassadou et Diomandou Dieri, a révélé une zoophagie exclusive d'An. rufipes qui a été abondant dans les récoltes de la faune résiduelle endophile et quasi absent des captures nocturnes sur appâts humains (Hamon et al., 1965; Konaté et al., 1994a; Faye et al., 1995b). Ces observations révèlent bien la stricte zoophilie des femelles de cette espèce.

Le grand cheptel, dont principalement les bovins, reste la source principale des repas sanguins pour cette espèce. Après les bovins, les femelles d'An. rufipes se nourrissent préférentiellement sur les équins.

\section{CONCLUSIONS}

A u Sénégal, les femelles des espèces du complexe An. gambiae, bien que très polyphages, se nourrissent préférentiellement sur homme dans toutes les localités prospectées. Leur comportement trophique a été principalement fonction de la disponibilité des hôtes. En milieu urbain de Dakar et de Pikine, l'homme constitue le seul hôte pour les femelles d'An. arabiensis, du fait de l'absence d'animaux. Il est également la source exclusive de repas de sang d'An. melas à Jifere, du fait de l'absence de tout bétail. Lorsque les femelles ont accès à la fois aux hommes et à un cheptel abondant, une fraction zoophage (plus ou moins importante selon les localités) est toujours notée. Les variations de l'IA s'expliquent en grande partie par celles des conditions d'accès à l'homme. Ainsi, dans la moyenne vallée du fleuve Sénégal, l'inaccessibilité des hôtes humains (due à l'emploi de moustiquaires) explique la forte zoophagie des espèces du complexe An. gambiae.

Dans les villages pratiquant à la fois l'agriculture et l'élevage, les mouvements saisonniers du bétail expliquent, pour une large part, la variation saisonnière des proportions des différents types de repas sanguins. Les résultats sur les IA d'An. gambiae et $A n$. arabiensis contrastent avec les observations qui font état d'une plus faible anthropophilie d'An. arabiensis. Les études effectuées au Sénégal ont montré que, lorsqu'ils étaient capturés au même moment, à la même place et par la même méthode d'échantillonnage, $A n$. gambiae et $A n$. arabiensis ont un comportement trophique similaire et présentent un indice d'anthropophilie, une proportion de repas mixtes et de femelles zoophages comparables. Pour ces dernières, le bovidé constitue l'hôte de premier choix. Lorsqu'il est absent, les équidés le plus fréquemment et les ovins/caprins dans une moindre mesure, constituent les sources de repas sanguins pour les femelles zoophages. 
Les femelles d'An. funestus ont été significativement plus zoophages dans le sud-est que dans le centreouest du pays. An. pharoensis, présent dans toutes les régions (en abondance dans le delta), se gorge essentiellement sur hôtes humains. Par contre An. rufipes, espèce très endophile, est strictement zoophile dans toutes les localités où elle est rencontrée.

\section{REMERCIEMENTS}

$\mathrm{N}$ ous remercions tous les techniciens du Service de Lutte Antiparasitaire, de l'ORSTOM, l'Université Cheikh Anta Diop et les habitants des villages d'étude pour leur assistance. Nous exprimons également nos remerciements au $\mathrm{D}^{\mathrm{r}}$ Didier Fontenille, du laboratoire de Zoologie Médicale de l'ORSTOM de Dakar, pour l'appui apporté à cette étude.

Ce travail a bénéficié d'un soutien financier du programme spécial PNUD/Banque Mondiale/OMS-TDR, de l'Institut Français de Recherche pour le Développement en Coopération (ORSTOM), du Ministère Français de la Coopération et du Développement, de SUMITOMO Chemical Co (Japon) et de l'Association des Universités Partiellement ou Entièrement de Langue Française (AUPELF).

\section{BIBLIOGRAPHIE}

Beier J.C., Perkins P.V., Wirtz R.A., Koros J., Diggs D., Gargan T. P \& Koech D.K. Blood meal identification by direct Enzym-Linked Immnosorbent Assay (ELISA), tested on Anopheles (Diptera: Culicidae) in Kenya. Journal of Medical Entomology, 1998, 25, 9-16.

Bruce-Chwatt L.J., Gockel C.W. \& Weitz B. A study of the blood feeding patterns of Anopheles mosquitos through precipitin tests. Bulletin of the World Health Organization, 1960, 22, 685-720

Bruce-Chwatt L.J., Garrett-Jones C. \& Weitz B. Ten years study (1955-1964) of host selection by anopheline mosquito. Bulletin of the World Health Organization, 1966, 35 (3), 405-439.

Carnevale P. \& Boreham P.F.L. Études des préférences trophiques d'Anopheles nili. Cahiers ORSTOM, série Entomologie médicale et Parasitologie, 1978, 16 (1), 17-22.

Carrara G.C., Petrarca V, Niang M \& Coluzzi M. Anopheles pharoensis and transmission of Plasmodium falciparum in the Senegal River delta, West Africa. Medical and Veterinary Entomology, 1990, 4, 421-424.

Chandler J.A., Boreham P.F.L., Highton R.B. \& Hill M.N. A study of the host selection patterns of the mosquitoes of the Kisumu area of Kenya. Transactions of the Royal Society of Tropical Medicine and Hygiene, 1975, 69, 415425 .

Coluzzi M. Heterogeneities of the malaria vectorial system in tropical Africa and their significance in malaria epide- miology and control. Bulletin of the World Health Organization, 1984, 62 (suppl.), 107-113.

Coluzzi M., Sabatini A., Petrarca V. \& Di Deco M.A. Chromosomal differentiation and adaptation to human environments in the An gambiae complex. Transactions of the Royal Society of Tropical Medicine and Hygiene, 1979, 73, 483-497.

Diatta M., Spiegel A., Lochouarn L. \& Fontenille D. Similar feeding preferences of Anopheles gambiae and Anopheles arabiensis in Senegal. Transactions of the Royal Society of Tropical Medicine and Hygiene, 1998, 92, 270-272.

Diop A. Épidémiologie du paludisme dans le delta du Saloum (Fatick, Sénégal) : rôle d'Anopheles melas dans la transmission. Thèse Doct. $3^{\text {e }}$ cycle de Biologie Animale ( $\mathrm{N}^{\circ} 38$ ), Faculté des Sciences et Techniques, Université Cheikh Anta Diop, Dakar, 1998, 117 p.

FAYE O. Contribution à l'étude des Anophelinae (Diptera : Culicidae) et de la transmission du paludisme dans la zone du barrage anti-sel de Bignona (Ziguinchor, Sénégal). Thèse Doct. $3^{\text {e }}$ Cycle de Biologie Animale, Université Cheikh Anta Diop, Dakar, 1987, 217 p.

FAYE O. Épidémiologie du paludisme dans les îles du Saloum. Rapp. Bourse AUPELF/UREF, 1997, 20 p

Faye O., Fontenille D., Herve J.-P., Diack P.A., Diallo S. \& Mouchet J. Le paludisme en zone sahélienne du Sénégal. 1. Données entomologiques sur la transmission. Annales de la Société belge de Médecine tropicale, 1993, 73, 21-30.

Faye O., Gaye O., Faye O. \& Diallo S. La transmission du paludisme dans des villages éloignés ou situés en bordure de la mangrove au Sénégal. Bulletin de la Société de Pathologie Exotique, 1994, 87 (3), 157-163.

Faye O., Konaté L., Fontenille D., Gaye O., Sy N., Hebrard G., Hervé J.-P. \& Touré Y.T. Variations saisonnières des populations d'Anopheles gambiae s.l. et transmission du paludisme dans un village de savane soudanienne du sud-est du Sénégal. Bulletin de l'Institut Fondamental d'Afrique Noire, Cheihk Anta Diop, Dakar, série A, 1995a, 48, 57-66.

Faye O., Gaye O., Fontenille D., Hebrard G., Konaté L., Sy N., Hervé J.-P., Touré Y., Diallo S., Molez J.-F. \& Mouchet J. La sécheresse et la baisse du paludisme dans les Niayes du Sénégal. Cahiers Santé, 1995b, 5, 299-305.

Faye O., Fontenille D., Gaye O., Sy N., Molez J.F., Konaté L., Hebrard G., Hervé J.-P., Troulllet J., Diallo S. \& MouCHET J. Paludisme et riziculture dans le delta du fleuve Sénégal. Annales de la Société belge de Médecine tropicale, $1995 \mathrm{c}, 75,179-189$.

Fontenille D., Faye O., Konaté L., Sy N. \& Collins F.H. Comparaison des techniques PCR et cytogénétique pour la détermination des membres du complexe Anopheles gambiae au Sénégal. Annales de Parasitologie Humaine et Comparée, 1993, 68, 239-240.

Fontenille D., Lochouarn L., Diagne N., Sokhna C., Lemasson J.-J., Diatta M., Konaté L., Faye F., Rogier C. \& TRAPE J.-F. High annual and seasonal variations in malaria transmission by anophelines and vector species composition in Dielmo, a holoendemic area in Senegal. American Journal of Tropical Medicine and Hygiene, 1997a, 56 (3), 247-253. 
Fontenille D., Lochouarn L., Diatta M., Sokhna C., Dia I., Diagne N., Lemasson J.-J., Ba K., Tall A., Rogier C. \& Trape J.F. Four years entomological study of the transmission of seasonal malaria in Senegal and the bionomics of Anopheles gambiae and A. arabiensis. Transactions of the Royal Society of Tropical Medicine and Hygiene, 1997b, 91, 647-652.

Gilies M.T. \& De MEILlon B. The anophelinae of Africa south of the Sahara (Ethiopian Zoogeographical region). Publications of the South Africa Institut of Medical Research, Johannesburg, 1968, 55, 343 p.

Gordon S.W., Tammariello R.F., Linthicum K.J., Wirtz R.A. \& DigoutTE J.-P. Feeding patterns of mosquitoes collected in the Senegal river basin. Journal of the American Mosquito control Association, 1991, 7 (3), 424-432.

Hamon J., Sales S., Coz J., Ouedraogo C.S., Dyemkouma A. \& Diallo B. Observations sur les préférences alimentaires des moustiques de la République de Haute-Volta. Bulletin de la Société de Pathologie Exotique, 1964, 57 (5), 1133-1150.

Hamon J., Coz J., Sales S. \& Ouedraogo C.S. Études entomologiques sur la transmission du paludisme humain dans une zone de steppe boisée, la région de Dori (République de Haute Volta). Bulletin de l'Institut Fondamental d'Afrique Noire, Cheibk Anta Diop, Dakar, série A, 1965, XXVII (3), 1116-1150.

Konaté L., Diagne N., Brahima K., Faye O., Legros F., Rogier C., Petrarca V. \& Trape J.-F. Biologie des vecteurs et transmission de Plasmodium falciparum, $P$. malariae et $P$. ovale dans un village de savane d'Afrique de l'Ouest (Dielmo, Sénégal). Parasite, 1994a, 1, 325-333.

Konaté L., Faye O., Diallo S. \& Ndir O. Le paludisme dans le Département de Dakar. I. Données entomologiques (résultats préliminaires). $4^{e}$ Congrès de la SOAP (0509/12/94), 1994b, Ouagadougou, Burkina Faso.

Lemasson J.-J., Fontenille D., Lochouarn L., Dia I., Simard F., Ba K., Diop A., Diatta M. \& Molez J.-F. Comparison of behavior and vector efficiency of Anopheles gambiae and An. arabiensis (Diptera : Culicidae) in Barkedji, a sahelian area of Senegal. Journal of Medical Entomology, 1997, 34 (4), 396-403.

MBAYE P.A. Impact des aménagements hydro-agricoles sur le paludisme. Étude menée dans des villages riverains du fleuve Sénégal et du lac de Guiers. Doctorat d'État, Faculté de Médecine, de Pharmacie et d'Odonto-Stomatologie ( $N^{\circ}$ 006), Université Cheikh Anta Diop, Dakar, 1997, 62 $\mathrm{p}$.

Mnzava A.E.P., Rwegoshora R.T., Wilkes T.J., Tanner M. \& CurTis C.F. Anopheles arabiensis and An. gambiae chromosomal inversion polymorphism, feeding and resting behaviour in relation to insecticide house spraying in Tanzania. Medical and Veterinary Entomology, 1995, 9, 316-324.

PASKEwITZ S.M. \& Collins F.H. Use of polymerase chain reaction to identify mosquito species of the Anopheles gambiae complex. Medical and Veterinary Entomology, 1990, 4, 367-373.

Petrarca V., Vercruysse J. \& Coluzzi M. Observations on the Anopheles gambiae complex in the Senegal River basin, West Africa. Medical and Veterinary Entomology, 1987, 1, 303-312.
Petrarca V., Beier J.C., Onyango F., Koros J., Asiago C., Koech D.K. \& Roberts C.R. Species composition of the Anopheles gambiae complex (Diptera : Culicidae) at two sites in western Kenya. Journal of Medical Entomology, 1991, 28, 307-313.

Robert V., Dieng H., Lochouarn L., Traoré S.F., Trape J.F., Simondon F. \& Fontenille D. La transmission du paludisme dans la zone de Niakhar, Sénégal. Tropical Medicine and International Health, 1998, 3 (8), 667-677.

Schubert J.H. \& Holdeman L.V. A modified precipitin technique for determining the source of mosquito blood-meals. American Journal of Tropical Medicine and Hygiene, 1956, 5 (2), 272-273

SharP L.B. \& LE Sueur D. Behavioural variation of Anopheles arabiensis (Diptera : Culicidae) populations in Natal, South Africa. Bulletin of Entomological Research, 1991, 81, 107110.

SNOW W.R. The attractiveness of some birds and mammals for mosquitoes in the Gambia (West Africa). Annals of Tropical Medicine and Parasitology, 1983, 77 (6 ), 641-651.

Tempelis C.H. \& Lofy M.F. A modified precipitin method for identification of mosquito blood meals. American Journal of Tropical Medicine and Hygiene, 1963, 12, 825-831.

Trape J.-F., Lefebvre-Zante E., Legros F., Ndiaye G., Bouganali H., Druilhe P. \& Salem G. Vector density gradients and the epidemiology of urban malaria in Dakar, Senegal. American Journal of Tropical Medicine and Hygiene, 1992, 47, 181-189.

VerCRUYSSE J. Étude entomologique sur la transmission du paludisme humain dans le bassin du fleuve Sénégal (Sénégal). Annales de la Société belge de Médecine tropicale, 1985, 65 (2), 171-179.

Vercruysse J. \& Jancloes M. Étude entomologique du paludisme humain dans la zone urbaine de Pikine (Sénégal). Cabiers ORSTOM, série Entomologie médicale et Parasitologie, 1981, 9, 165-178.

White G.B. Blood feeding habits of mosquitoes in the South Pare district of Tanzania ten years after cessation of the dieldrin residual spraying campaign. East African Medical Journal, 1971, 48, 122-134.

White G.B., Magayuka S.A. \& Boreham P.F.L. Comparative studies on sibling species of the Anopheles gambiae Giles complex (Diptera : Culicidae): bionomics and vectorial activity of species A and species B at Segera, Tanzania. Bulletin of Entomological Research, 1972, 62, 295-317.

White G.B. \& Rosen P. Comparative studies on sibling species of the Anopheles gambiae Giles complex (Diptera : Culicidae). II. Ecology of species $\mathrm{A}$ and $\mathrm{B}$ in savana around Kaduna, Nigeria, during transition from wet to dry season. Bulletin of Entomological Research, 1973, 63, 61325.

Reçu le 15 janvier 1998 Accepté le 16 février 1999 